\title{
Estudio del comportamiento porcino: una mirada etológica sobre la producción porcina
}

\author{
Porcine behavior study: an ethological view on swine production
}

\author{
Arroyo P $^{*}$, Ferrari HR ${ }^{2}$, ANtonini AG ${ }^{1}$
}

1. Instituto de Genética Veterinaria, Facultad de Ciencias Veterinarias. 2. Cátedra de Etología, Facultad de Ciencias Naturales y Museo. Universidad Nacional de La Plata

* Correo electrónico de la autora de contacto: parroyo@fcv.unlp.edu.ar

\begin{abstract}
Resumen
El conocimiento del comportamiento permite mejorar el manejo de los animales utilizados en producción y optimizar su bienestar. La disciplina de las ciencias biológicas que aborda este tema es la etología. Basada en la teoría de la evolución, pone énfasis en cuatro tipos de explicaciones, conocidas como las " 4 preguntas de Tinbergen”. ¿Son estos cuatro abordajes útiles para la etología aplicada a la producción de porcinos? ¿Las investigaciones en etología pueden aportar a la mejora en las condiciones de crianza? Y, fundamentalmente, ¿̇son relevantes para el bienestar animal? Algunas prácticas realizadas de manera "intuitiva" o "empírica" hoy pueden ser identificadas como "la ayuda silenciosa" que la etología ha aportado a cada etapa productiva desde el inicio de la utilización de la cría de animales domésticos, particularmente cuando esta comienza a realizarse de manera intensiva. Esta ayuda silenciosa generó un conjunto de saberes específicos que, paradójicamente, no se relacionan con esta disciplina. Proponemos aquí darle voz a esta ayuda, identificando los distintos desarrollos etológicos implicados en la producción porcina.
\end{abstract}

\section{Palabras clave}

producción porcina, etología, bienestar animal

\begin{abstract}
The comprehension of behavior allows to improve the animal handling used in production and optimize their welfare. The discipline of the biological sciences that addresses this issue is ethology.

Based on the theory of evolution, it emphasizes four types of explanations, known as the "four questions of Tinbergen". Are these four approaches useful for the applicated ethology in swine production? Can research on ethology contribute to better breeding conditions? Fundamentally, are they relevant for animal's welfare? Today, some practices performed in an "intuitive" or "empirical" way can be identified as "the silent help" in which ethology has contributed in each productive stage since the beginning of the use of the domestic animals breeding, particularly when it turns into an intensive manner. This silent help generated a combination of specific knowledge which paradoxically do not relate with this discipline. It is proposed here by giving the voice to this help, identifying the different ethologist developments in swine production.
\end{abstract}

\section{Key words}

pig production, ethology, animal welfare

Fecha de recepción: 05/10/2017

Fecha de aprobación: 26/12/2017
ANALECTA VeT 2018; 38(1): 23-32

Impresa ISSN 03655 14-8 Electrónica ISSN 1514-2590

doi.org/10.24215/15142590eo22 


\section{Introducción}

El conocimiento del comportamiento permite mejorar el manejo de los animales utilizados en producción y optimizar su bienestar. La disciplina de las ciencias biológicas que aborda este tema es la etología. Basada en la teoría de la evolución, hace énfasis en cuatro tipos de explicaciones, conocidas como las "cuatro preguntas de Tinbergen", las que se refieren a la filogenia, la ontogenia, la fisiología y el efecto del comportamiento en la supervivencia y la reproducción. Originadas a mediados del siglo pasado (Tinbergen, 1963), si bien se han sometido a periódicos análisis y revisiones, (Alcock, 2001; Curio, 1994; Dewsbury, 1999; Duncan, 1995; Hogan, 2015; Stamps, 2003) se siguen empleando con su formulación inicial.

Por filogenia entendemos la historia evolutiva de las características comportamentales en estudio. Esto no es una mera relación histórica, sino que se refiere a los cambios que fueron llevando de una conducta a otra hasta llegar al rasgo actual; en términos de producción esto significa que lo que estamos viendo es el producto de una larga historia de interacciones entre una población y un ambiente ancestral de selección que ya no está presente. Muchas de las problemáticas de bienestar animal se deben a ese desajuste entre las características y el ambiente, las cuales son más fáciles de entender si conocemos esa historia evolutiva.

La ontogenia estudia los cambios de desarrollo en la vida de un individuo de ese mismo rasgo. En la etología clínica de los animales domésticos los periodos sensibles en el desarrollo son los momentos cruciales de la ontogenia que determinarán la futura relación individuo-entorno. Para la medicina veterinaria, los mecanismos fisiológicos de regulación del comportamiento están amplia-mente estudiados y son utilizados en la práctica profesional.

La cuarta pregunta, que hace referencia a los efectos, pone énfasis en los cambios en la supervivencia y en la reproducción generados por un rasgo conductual. En el caso de los animales de producción, estos dos aspectos están regulados por los criadores en función de los objetivos productivos, pero las conductas de los animales siguen respondiendo a los efectos que tenían en el ambiente ancestral de selección.

¿Son estos cuatro abordajes útiles para la etología aplicada a la producción en porcinos? ¿Las investigaciones en etología pueden aportar a la mejora en las condiciones de crianza? Y, fundamentalmente ¿son relevantes para el bienestar animal?

Tomando la definición de Donald Broom (1991), que caracteriza el bienestar animal como el estado de un individuo en sus intentos por afrontar su ambiente, y entendiendo que la relación con el medio ambiente es mediante el comportamiento, queda establecida la relevancia de la etología con respecto a la importancia de este problema.

Las cuatro preguntas de Tinbergen, en este contexto, son las explicaciones que el etólogo da a la forma en que un animal afronta su ambiente.

A mediados del siglo pasado comenzó el interés social por el bienestar de los animales, con el consecuente desarrollo de investigaciones que tenían esta inquietud como eje. Respondiendo inicialmente a un imperativo ético que emergía del público, al conocer este las condiciones de producción, fue derivando en la formación de un campo transdisciplinar. En la actualidad incluye estudios de psicología comparada, antropología, sociología y medicina veterinaria y todos contextualizados en desarrollo de filosofía $\mathrm{y}$, en algunos casos, de religión.

$\mathrm{Si}$ bien el bienestar animal debe ser abordado interdisciplinariamente, el Médico Veterinario tiene un rol fundamental en la implementación de estrategias y métodos de trabajo. Por esa razón es necesario que en el proceso de formación de los futuros profesionales se incluyan contenidos referidos al comportamiento, en particular de las principales especies de producción. Estos contenidos tomarán principalmente la forma de respuestas a las cuatro preguntas y estas respuestas empezarán por organizar, de manera diferente, el conocimiento actual, para luego proponer nuevas direcciones de investigación. Actualmente, los profesionales dedicados a la producción animal han comenzado a identificar la importancia de reconocer la etología como una herramienta útil en la definición de estrategias de manejo en los diferentes sistemas de producción, hasta el extremo de sugerir la inclusión de cursos de etología teórica o pura en las distintas carreras de veterinaria.

Algunas prácticas realizadas de manera "intuitiva" o "empírica" hoy pueden ser identificadas como "la ayuda silenciosa" que la etología ha aportado a cada etapa productiva desde el inicio de la utilización de la cría de animales domésticos, particularmente cuando ésta comienza a hacerse de manera intensiva. Esta ayuda silenciosa generó un conjunto de saberes específicos que, paradójicamente, no se relacionan con esta disciplina. Proponemos aquí darle voz a esta ayuda identificando los distintos desarrollos etológicos implicados en la producción porcina.

\section{Filogenia: implicancia en la producción porcina}

Toda especie doméstica puede reconocerse como producto de tres procesos evolutivos. En un principio, los ancestros silvestres fueron moldeados mediante los procesos de selección natural y selección sexual, como resultado de lo cual las poblaciones desarrollaron y conservaron potencialidades genéticas orientadas a su supervivencia y 
reproducción. En algunos casos, en algunas poblaciones ocurrió un proceso denominado "autodomesticación": las condiciones ambientales favorecieron una baja en las conductas de agresión llevando a una mayor tolerancia entre los individuos; se cree que fue con individuos de estas poblaciones que se generaron los actuales animales domésticos.

La domesticación es entendida como el proceso de selección, por parte de los humanos, de los individuos mejor adaptados a las necesidades de los productores. Esto llevó a cambios genéticos en las poblaciones, expresados en cambios en las frecuencias de alelos y, consecuentemente, de los genotipos que ellos determinan. A diferencia de lo que ocurrió con la selección natural, en la domesticación los cambios se orientaron principalmente a beneficiar a las personas, poniendo a la supervivencia y a la reproducción de los animales a cargo del productor.

Mientras que para un animal silvestre el ser humano, por lo general, es o presa o predador, los cambios ocurridos durante la domesticación hacen que las poblaciones que lo sufren sean a veces totalmente dependientes de la conducta humana. Los animales domésticos son más gregarios, menos cautelosos, más juguetones, presentan precocidad sexual, son más receptivos y el ambiente de estos animales es más estable y predecible. La complejidad cognitiva no ha pasado por cambios especiales relacionados con la domesticación. En el cerdo este proceso comenzó hace aproximadamente 9.000 años en el sudoeste asiático. De allí se expandió hacia India, luego al este de Asia y finalmente a Europa.

Gracias al estudio de ADN mitocondrial, Larson et al., en 2005, lograron identificar los principales centros de domesticación del ganado porcino, uno en Asia y dos en Europa (uno en la Península Ibérica, (sur de España y Portugal) y otro en la región del Mediterráneo (sur de Francia, Italia, Grecia, Malta, Austria, Hungría y Bulgaria).

Debido a una alta proximidad con las personas y a su consecuente selección, se evidencian diferencias en el comportamiento entre las diferentes razas, como por ejemplo que la raza Meishan china y la Large White europea difieren en su comportamiento al amamantar (ManierSalaün et al., 1991). Hohenshell et al., (1996), concluyeron que la Meishan y las híbridas de 2 razas europeas, Large White y Landrace, difieren en las frecuencias de las pautas "arrodillada" y "sentada".

Por su parte, los porcinos que llegaron a América fueron de origen europeo, principalmente de la Península Ibérica, pero también del tronco mediterráneo, y se aparearon de manera no controlada por el ser humano, hasta mediados del siglo XIX. Durante los siguientes 100 años se introdujeron otras razas para absorber al llamado ganado porcino criollo, resultado de los cruzamientos de las primeras razas que llegaron. A la Argentina los cerdos ingresaron por 3 vías: por el Río de La Plata en 1557, desde Paraguay en 1573 y finalmente desde el alto Perú. Estos animales se criaron como ganado cimarrón, y se explotaron estas líneas hasta 1960, cuando se importaron reproductores Landrace desde Alemania e Inglaterra (Revidatti, 2009). Debido a sus diferentes orígenes y métodos de expansión de este ganado, podemos observar distintos tipos de temperamento en las razas puras, pero a su vez reconocemos la alta frecuencia de cruzamientos interraciales y similitudes en el comportamiento de las principales razas de explotación en nuestro país, gracias a que sus orígenes se han ido entrelazando a lo largo de la historia de domesticación y cría.

Todo lo anterior puede considerarse una versión resumida de la historia evolutiva de estos animales. Entonces, la conducta que debemos contemplar a la hora de producir ha sido originada por el pasado remoto como silvestres, el pasado próximo como domesticados y la situación actual como cruza entre las distintas razas. Las situaciones de bienestar animal con impacto en la producción son producidas por las diferencias entre el ambiente actual (plasmado en las instalaciones) y el ambiente ancestral de selección; las conductas de las razas responden más al segundo que al primero.

\section{Ontogenia: historias de vida y producción}

Un estudio comparativo entre cerdos silvestres y domésticos concluyó que las diferencias en sus capacidades cognitivas se deben a la ontogenia (Albiach-Serrano et al., 2012). Los cerdos frecuentemente se escapaban de los humanos volviéndose ferales y se mezclaban con las poblaciones locales. Esta falta de aislamiento genético completo puede haber mantenido la homogeneidad cognitiva en ambas poblaciones. Es posible considerar que la domesticación haya empobrecido su habilidad para acoplar con el ambiente físico $\mathrm{y}$, por lo tanto, las diferencias observadas no pueden ser atribuibles a procesos de cambios evolutivos. Por lo tanto, estas diferencias no responden al pasado evolutivo de la especie, sino al desarrollo actual de los individuos. En casos como este, la manipulación del ambiente y de la relación humano-animal puede llevar a expresiones comportamentales que faciliten el proceso evolutivo, por ejemplo, el acostumbramiento de los animales al contacto con humanos.

\section{Aspectos causales del comportamiento}

Entre la multiplicidad de factores que determinan una conducta existen efectos indirectos que suelen no ser tenidos en cuenta. Por ejemplo, la manera de percibir los colores o las formas determina, en parte, el comportamiento, es decir las variaciones filogenéticas tienen impacto en la manera de expresar un evento observable. 
De esta manera decimos que los compartimientos típicos de cada especie han evolucionado. En el trabajo de Finlay et al., (2011) se menciona la diferencia en el tamaño del cerebro entre las especies de mamíferos, en sus maneras de percibir formas $\mathrm{y}$, como consecuencia, los distintos comportamientos. Estas percepciones responden a mecanismos y estructuras específicos de cada especie; hablamos entonces de mundo perceptivo o Umwelt.

Prestaremos aquí atención a un aspecto de esta regulación: la percepción. Los órganos de los sentidos no son ventanas, sino elementos sensibles a determinadas cualidades del entorno. Cada especie tiene los suyos propios, que le son específicos, y que han evolucionado en el ambiente ancestral de selección: son, en cierta forma, el producto de la historia ecológica de la especie, adaptados a maximizar la eficiencia para ese tipo de vida, en un ambiente determinado (Lorenz, 1995).

La percepción, a su vez, es producida por el cerebro (Lorenz, 1995). La dinámica neuronal es el resultado de la suma espaciotemporal de estímulos. Percibir es computar, y ese cómputo es específico de especie, porque el cerebro que procesa los estímulos, y los órganos de los sentidos que los generan, son específicos de especie. A esto lo denominamos Umwelt: mundo sensorial y perceptual propio, característico de una especie (Thorpe, 1982). Así, cada individuo percibe el mundo de una manera que le es propia, y como la conducta la dirige hacia lo que percibe, vive en un mundo propio. En el caso que nos ocupa, en los cerdos se distingue el olfato. Probablemente existan feromonas con mensajes muy específicos. Podría haber una relacionada con la sumisión. Si se colocan gotas opacas en los ojos de los animales no cambia la conducta social, pero se observan cambios cuando se produce anosmia (Jensen, 2004). Sin embargo, existen señales visuales específicas en jabalíes y cerdos domésticos, como por ejemplo posturas de las orejas, cola o cuerpo. Orejas hacia atrás sobre el cuerpo comunican temor, cola rígida y hacia arriba, peligro y cola caída, sumisión. También emplean posturas corporales: inclinar la cabeza a un lado es sumisión y arquear el lomo es amenaza.

La visión es también modulada por su anatomía, ya que detrás de la cabeza tienen una zona ciega, que abarca un ángulo de 15 grados a cada lado del eje del cuerpo y, por delante, una de visión binocular, de 25 grados a cada lado del eje del cuerpo (Grandin, 1980).

El oído cumple un papel en la estructura regulatoria. Tienen un repertorio de llamados vocales. Uno de alarma, cuando el individuo está en peligro, por ejemplo, al oír a un perro. Responden repitiendo la llamada, permaneciendo muy quietos o huyendo. Emiten gruñidos de contacto, chillidos de sumisión, llamadas a la madre y gemidos de lactación (Jensen, 2004). Entonces, el mundo del cerdo no es el nuestro ni el de un perro: es el que sus sentidos pueden registrar y su cerebro procesar.

\section{Efectos en la supervivencia y la reproducción}

En la fase de lactancia, o etapa de maternidad, la etología nos ha explicado muchas de las observaciones que podemos realizar, tanto sobre la hembra como sobre los lechones, o las interacciones entre estos.

En las primeras 24 horas postparto, luego de haber calostrado, se realiza una práctica denominada adopción cruzada, en la cual se equiparan en cantidad y peso los lechones de las camadas de las distintas hembras que parieron.

Debe ser en este periodo, debido al troquelado o impronta madre-cría, periodo ventana en el cual se realiza el reconocimiento de uno por parte del otro en ambos sentidos, el cual será permanente. Constituye el proceso por el cual ciertos estímulos se asocian para provocar determinadas conductas (Lorenz, 1937).

Durante esta etapa se observa la comunicación entre la madre y la cría (entendiendo a la comunicación como el proceso por el cual un actor influencia a un reactor, según Tinbergen -1939-, mediante señales). Dicho proceso se pone en evidencia mediante el cambio de frecuencia del sonido emitido por la hembra, obteniendo como resultado que la cría adopte el comportamiento de mamar (Jensen, 2004).

La competencia de los lechones por las tetas craneales para mamar puede recibir una lectura desde la etología. Básicamente, desde la así llamada cuarta pregunta de Tinbergen: ¿cuales son los efectos en la supervivencia y la reproducción de esta conducta? Básicamente, lo que se analiza es que cada lechón se "apega" a una teta, y se disputan las craneales, observándose lechones más grandes en las mamas torácicas, y algunos en 2 mamas (Hartsock \& Graves, 1976). Esto se hace en el contexto de una así llamada "carrera armamentista": nacen con 8 dientes agudos orientados hacia lateral ya en condición de infringir cortes durante las interacciones agonísticas con otras crías (Fraser et al., 1995).

Existen diversos argumentos que intentan explicar estas observaciones. En primer lugar, desde el punto de vista nutricional, las tetas craneales proveerían más leche, en especial, más calostro. Esto resulta no ser así (Fraser-Rushen, 1992; Orihuela-Solano, 1995). En segundo lugar, las posibilidades de supervivencia, ya que, en las tetas caudales, las crías pueden ser aplastadas al sentarse la madre (Fraser 1990; Fraser et al., 1995). En tercer lugar, la defensa de las tetas de los "ladrones de leche", ya que permite expulsar a los individuos de otra camada que tratan de mamar de una hembra que no es su madre (Fraser, 1991). Un estudio muestra que los desplazados no pierden peso y los desplazadores no lo ganan (Illman et al., 2007). Esta es, entonces, un área pendiente de 
investigación. En cuarto lugar, las hembras paren, en promedio, un $20 \%$ más de crías de las que sobrevivirán, entendiéndose la competencia como una estrategia para disminuir la cantidad de lechones con el fin de no comprometer el ciclo reproductivo siguiente. Esto, en el contexto de la producción porcina actual, se ve compensado en la mayor cantidad de mamas que poseen las cerdas y en el manejo en el parto y periparto que aumenta la supervivencia de los lechones (Fraser, 1991). Por último, otra teoría respecto a la funcionalidad de la presencia de los dientes filosos en los neonatos es que estos sirven para romper la placenta, aspecto irrelevante si consideramos que otras especies no los poseen y son capaces de liberarse de las membranas placentarias (Fraser \& Thompson, 1991).

Continuando con el acto de amamantamiento, se puede analizar el masaje de las mamas por parte de los lechones. Dostalkova et al., (2002) desarrollaron un modelo matemático con el fin de entender la co-evolución o evolución conjunta del masaje de tetas y la provisión de leche. En este trabajo plantearon cómo el aumento en la performance relativa de un individuo tiene una relación inversa con la performance grupal, por lo que la selección basada en el grupo disminuye los comportamientos competitivos. De esta manera, y debido a los objetivos de los programas de selección, la evolución podría conducir a disminuir el comportamiento de "súplica" (masaje de mamas por parte de los lechones). Son dos aspectos de esta interacción, la madre y las crías. En el caso de la madre, la respuesta a la conducta de las crías tiene efectos en la reproducción y, en el caso de las crías, en la supervivencia; es decir, todos, madre e hijos tienen el mismo objetivo: que estos últimos sobrevivan. Más tarde, se observa el llamado "conflicto madrecría”, en el que los requerimientos de los lechones comienzan a dejar de ser prioridad para los progenitores. Para ejemplificar esto mencionaremos el hecho que cuanta más cantidad de leche produce una hembra, más se compromete su futuro reproductivo, ya que se prolongará el intervalo destete-celo. Es por esto que el acto de amamantamiento es finalizado por la hembra en el 30-50 \% de los casos en la primera semana (Spinka \& Algers, 1995).

\section{Necesidades comportamentales y comporta- miento innato. Un aporte de la etología al problema del bienestar animal}

Respecto del comportamiento, una herramienta de análisis pertinente y sumamente útil es el concepto de necesidad comportamental, ya mencionado por Thorpe en el informe Brambell (1965). Veamos, por ejemplo, el análisis de Jensen \& Toates (1993).

Según estos autores, este concepto puede rastrearse hasta el escrito presentado por Thorpe al comité Brambell (1965). Él acuñó la idea de que los animales tienen necesidades comportamentales, además de las más obvias necesidades fisiológicas a las que el comportamiento atiende. Este concepto se basa en las teorías de Lorenz (1974) que plantea que la energía específica de acción puede crecer dentro del animal si este es privado de los estímulos liberadores (llave) relevantes.

En el modelo psicohidráulico de Lorenz, el cual explica el "comportamiento instintivo", el autor menciona cómo ciertos mecanismos, llamados estímulos llave, actúan sobre los mecanismos desencadenantes innatos, los cuales dispararán las pautas fijas de acción, que son aquellas que se dan siempre de la misma manera. Mediante este proceso explica cómo, al transcurrir cierto tiempo sin que se desarrolle una pauta, aumenta la posibilidad para que esta pauta ocurra sin su estímulo llave. De esta manera, la energía específica para esta acción se va acumulando hasta "rebalsar", es decir, se ejecutará la pauta aún en ausencia del estímulo y ocurrirán los llamados "disparos al vacío". En otras palabras, se observará la pauta carente de contexto. Un ejemplo de esto puede observarse en la etapa de engorde de la producción porcina actual, durante la cual se observa la succión de las colas por parte de sus congéneres. En este fenómeno, la pauta de succión se "dispara" aún en ausencia del estímulo "pezón". De tal manera, la separación de la madre constituye una situación estresante para los lechones, y la ausencia de las tetas maternas se establece como el estímulo principal que provoca el acrecentamiento de la probabilidad de ocurrencia de la succión, y uno de los factores más importantes en el origen de la caudofagia y la succión de parte de las instalaciones.

Si bien el modelo "psicohidráulico" es simple e intuitivo, muchas de las interpretaciones rea-lizadas con este método se pierden con mucha frecuencia en los modernos modelos motivacionales que solamente tienen en cuenta la necesidad fisiológica como única fuente del estímulo. Hughes \& Duncan (1998) afirman, en forma explícita, que el concepto de "necesidad" etológica puede ser clarificado solamente si se lo puede integrar satisfactoriamente en el contexto de un modelo motivacional.

En el presente trabajo se identifica una necesidad como un estado que, si no es atendido, causa sufrimiento a un animal que se evidencia como disturbios comportamentales, con un incremento en el riesgo de enfermedades y/o un perfil hormonal compatible con estrés. El estado motivacional es un estado del sistema nervioso. Algo que tiende a perderse en los modelos es la incorporación expresa de retroalimentación positiva, lo que lleva a cierta persistencia en la liberación del comportamiento.

Si dejamos de lado los modelos basados en energía, se puede hacer una serie de sugerencias sobre las causas, basadas en ellos: 
1. un comportamiento es liberado por una combinación de factores internos y externos,

2. cuando un comportamiento se realiza, después de un período inicial de retroalimentación positiva, la motivación se va debilitando progresivamente y el comportamiento se detiene, con independencia de sus consecuencias,

3. durante la no realización de un acto específico la motivación para ese acto se incrementa gradualmente.

Algunos autores han dividido las motivaciones en aquellas que contribuyen a la homeostasis, por ejemplo alimentarse, y aquellas que no, por ejemplo el apareamiento. Se ha argumentado que la primera clase está internamente motivada, mientras que la última lo está externamente; otros proponen que la teoría de la motivación por incentivos puede explicar una gran cantidad de resultados y permite que el sexo y la agresión sean discutidos en el mismo contexto que el comer y beber (Hughes \& Duncan, 1998).

En las ramas aplicadas de la etología que se ocupan del bienestar animal, el modelo de Wiepkema (1987) ha ganado influencia. De acuerdo con éste, los animales monitorean continuamente el estado del mundo externo (Istwert) y lo comparan con el Sollwert (una representación interna del estado deseado). Cuando hay una discrepancia, se realiza una acción para disminuirla. Cuando la discrepancia disminuye, el animal experimenta un sentimiento de placer.

Como en el modelo de Lorenz, se pueden deducir algunas características de los modelos homeostáticos, incluyendo el de Wiepkema (1987):

1. el comportamiento es liberado cuando el valor de una variable de control se aparta de un punto fijo, un óptimo de equilibrio,

2. el comportamiento apaga su motivación reduciendo la discrepancia entre los valores comparados,

3. cuando no se aparta la variable medida de su valor de referencia, el comportamiento no se libera.

En las versiones posteriores, Lorenz cambió levemente su idea de la motivación como un estado interno. Generalmente se utiliza la expresión "necesidades comportamentales" para describir la necesidad de realizar un patrón de comportamiento específico, cualquiera sea el ambiente, incluso si la necesidad fisiológica a la que el comportamiento atiende está satisfecha.

Esto significa que la realización del comportamiento que constituye una necesidad es motivacionalmente diferente de otros comportamientos en el repertorio del animal. Se separan "necesidades" de "lujos". Esto lleva a la "aproximación por el catálogo", donde el objetivo de la investigación es producir una lista de expresiones comportamentales especie-específicas que deben permitirse cuando el animal está en cautiverio.

Esta definición no aporta al conocimiento de las verdaderas motivaciones en el comportamiento y hace poco por el bienestar de los animales. Por un lado, empezando por Thorpe, están los que opinan que la aproximación de Lorenz es la manera más fructífera de contemplar la motivación. Por otro, hay autores que rechazan la existencia de un crecimiento interno de la motivación, apoyándose en modelos como el de Wiepkema (1987). Por ejemplo, Baxter (1983) propone el concepto de "necesidad psicológica" como una alternativa, significando que los animales tienen objetivos para sus actividades y, si estos pueden ser alcanzados sin que el animal actúe, el comportamiento nunca es liberado (disparos al vacío). Son dos posiciones extremas, en un continuo.

Hay ciertas actividades que son casi enteramente dependientes de fluctuaciones de estados internos para su desarrollo, como alimentarse, anidar y picotearse las plumas, mientras que otras dependen más exclusivamente de variaciones de estados externos, por ejemplo, comportamiento agonístico y respuestas antipredador. Hughes \& Duncan (1998) expresan: "Cuando el comportamiento es normalmente incitado por la presencia de factores externos, entonces la cuestión de la necesidad comportamental, obviamente, no aparece; si un fenómeno tal como una necesidad existe, entonces debe aparecer bajo la forma de patrones de comportamiento motivados principalmente por actores internos”.

Entonces, una necesidad comportamental debe: internos

1. ser causada principalmente por actores

2. su tendencia debe crecer gradualmente, en la medida que no es realizada,

3. la sola realización del comportamiento origina recompensa; el animal tratará de realizar el patrón motor con independencia del estado del ambiente y de las consecuencias funcionales de la actividad.

La necesidad de realizar el comportamiento debe también ser independiente del tipo de ambiente en el que el animal vive. Prohibir su realización se asociará con bienestar negativo. Sin embargo, prohibir otros comportamientos, que no cumplen estos criterios, puede ser seguro desde el punto de vista del bienestar.

Cuando comenzamos a mirar en detalle lo que parecen ser sistemas de comportamiento simples, encontramos que emerge una compleja imagen que sería errado categorizar en clases dependientes de, por ejemplo, una supuesta dicotomía entre factores internos y externos. Como se verá, esto lleva a los autores a la conclusión que no es posible clasificar algunos de 
los comportamientos como necesidades por comparación con otros. Se examinarán los tres criterios vistos antes, para mostrar que son demasiado simplificados para aportar a una teoría general de las necesidades comportamentales.

Uno de los casos investigados tiene que ver con producción en porcinos: la construcción del nido en cerdas. En un intento por distinguir los efectos de los dos tipos de factores, Jensen (1986, 1993) estudió la realización de las actividades de construcción del nido en cerdas en corrales libres de estímulos relevantes (corrales de concreto), comparando con cerdas en corrales similares pero enriquecidos, con cama que les permite hocicar y con paja en una canasta, para usar como material de nido.

No hubo diferencia en la cantidad de la actividad de nidificación vista en ambos grupos. Sin embargo, las cerdas en ambientes con pocos estímulos desarrollaron sólo los comportamientos correspondientes a la primera parte de la actividad (husmear, hociquear, patear) y no hicieron ningún intento de realizar en vacío las conductas de colocar u ordenar el material.

Así, no habría un "impulso nidificador" unitario. Por supuesto, esto puede significar que la nidificación constituye un complejo de dos procesos motivacionales diferentes: unos internos y otros externos. Tal vez la primera parte del patrón pueda calificar como necesidad (Hughes \& Duncan, 1998).

Una interpretación probable es que el incremento en la prolactina dispara un estado motivacional en el que dominan las tendencias a nidificar. Este estado luego se asocia con una representación de logro $\mathrm{y}$, al mismo tiempo, dispara la primera parte del programa motor. Si la cerda no alcanza algo que sea satisfactorio, que disminuya la diferencia Istvert-Sollwert, como dice Wiepkema, la siguiente parte del patrón no comienza.

El anidamiento en las cerdas claramente refleja una necesidad, pero sería un error considerar que eso constituye solamente realizar un cierto patrón motor en cualquier ambiente.

El hecho de que a las cerdas se les pueda impedir nidificar les provoca el desarrollo de comportamientos anormales, como estereotipias, lo que indicaría que el comportamiento está asociado a una necesidad.

Una conducta necesaria en el momento de la inseminación de hembras es su inmovilidad, que en su estado natural es simplemente un reflejo como respuesta a la monta del verraco. Reproducir esta conducta en ausencia del macho requiere establecer estrategias adecuadas, como por ejemplo la utilización de "mochilas" (dispositivo similar a un arnés con peso en los laterales, que se coloca sobre el dorso-lomo de la reproductora) que simulan el peso del macho sobre la hembra. También es necesaria la presencia de un macho "celador" que al segregar feromonas y emitir sonidos contribuye a la expresión de la conducta deseada.
En el caso de los padrillos, para realizar las extracciones seminales, se hace uso de un caballete o súcubo, que actuará como estímulo desencadenante de la conducta de monta, y esta pauta es, a su vez, reforzada de manera positiva, es decir, se los "premia". Skinner (1974) propone que "una conducta al obtener una consecuencia reforzante tiene más probabili-dades de ocurrir nuevamente en circunstancias similares".

\section{Una lectura etológica de las estereotipias y el enriquecimiento ambiental}

Bajo ciertas circunstancias los animales confinados desarrollarán estereotipias, las cuales constituyen comportamientos repetitivos, constantes en la forma y sin propósito obvio en el contexto, como por ejemplo el mordido de barrotes y el masticar al vacío (en algunos casos denominados animales "tragadores de aire"). Dentro de las alteraciones más notorias del comportamiento se encuentra el mordido de las colas de los congéneres. Todo esto puede ser entendido como necesidades comportamentales que el ambiente canaliza transformándolas en conductas dañinas para los individuos y su entorno. El hecho de que sean motivaciones intrínsecas las hace particularmente difíciles de manejar, siendo una de las formas más utilizadas para tratarlas mediante el enriquecimiento ambiental.

El enriquecimiento ambiental consiste en el proceso por el cual se modifica el ambiente de los animales con el objetivo de que aparezcan conductas nuevas y poder de esta manera mejorar el bienestar animal; puede llevarse a cabo de distintas formas y, para eso, se lo clasifica en 5 tipos (Hoy et al., 2010):

1. alimentario, en el cual se varía el alimento o este es presentado de una forma diferente. La presentación del alimento ad libitum y particulado reduce al mínimo el tiempo destinado a su búsqueda, captura y procesa-miento (haciendo referencia a las distintas etapas que constituyen el proceso de forrajeo), dejando tiempo libre para desarrollar las alteraciones antes mencionadas,

2. social, mediante el cual permitimos realizar interacciones con otro individuo de la misma especie, o incluso con los humanos (este tipo es difícil de aplicar a los reproductores en el actual tipo de alojamiento de los establecimientos intensivos confinados),

3. del hábitat. En este tipo de enriquecimiento se modifica parte de su recinto o alojamiento, por ejemplo, alojando a las hembras reproductoras en corrales grupales con el posterior traslado a los galpones de maternidad,

4. sensorial. Se les presentan a los animales nuevos olores, sonidos y/o texturas novedosas que estimulen sus sentidos, por ejemplo, cadenas, 
objetos colgantes o juguetes, que sean masticables, deformables y destruibles, entre otros,

5. cognitivo. En este caso se le brinda al animal la posibilidad de resolver un problema. El cerdo posee una gran necesidad comportamental respecto a la conducta de exploración, por lo que este enriquecimiento es uno de los aplicados más frecuentemente, por ejemplo, mediante la utilización de camas de paja. La manera de arribar al éxito o al fracaso de esta medida es registrando, o no, pautas nuevas de conducta en los animales y, de esta manera, mejorar su bienestar animal.

En el contexto de las necesidades comportamentales el enriquecimiento es una intervención realizada teniendo en cuenta el mundo perceptivo de una especie para propiciar el reencauzamiento de las conductas que generan el problema.

\section{Conclusiones}

Las cuatro preguntas de Tinbergen actúan a modo de paradigma, en el sentido que Lakatos (1983) da a ese término: el entramado conceptual que permite distinguir los objetos y métodos de una ciencia. Una vez definido un marco conceptual como este, desde él se generan las investigaciones y reflexiones que transforman la teoría.

Las prácticas médicas, en cambio, se orientan a transformar las condiciones de existencia: su finalidad no es generar teoría, sino aplicarla. Por supuesto, una misma persona puede a la vez generar teoría y aplicarla; ambos dominios cognoscitivos están conectados y son diferentes en estructura y procesos. En las disciplinas médicas aplicadas a la producción, el marco teórico de la etología cumple simultáneamente varias funciones. La más evidente es actuar como contramedida para el antropomorfismo: al situar las explicaciones en el contexto de la teoría de la evolución, dificulta o impide que transfiramos a ellas los mecanismos y motivaciones humanas.

Como corolario de lo anterior, los seres son vistos como el producto de tres procesos (su evolución, su desarrollo y su fisiología) lo que hace que sus características conductuales (o de otro tipo) respondan a tres dinámicas diferentes, que generan orden en el acoplamiento individuoentorno, orden cuya comprensión lleva a una mejor intervención. Esta propuesta de un orden en tres escalas temporales, que se refleja en una consecuencia ecológica (los efectos en la supervivencia y la reproducción se manifiestan en el ambiente) nos lleva a la problemática del bienestar animal. Es en este contexto que el uso de las cuatro preguntas de Tinbergen se inserta en el ejercicio de la medicina veterinaria.

El hecho de redefinir al ser vivo como algo más que un mecanismo, y de explicarlo por su triple historia de cambios en su relación con el ambiente, permite prestar atención a los desacoples que antes no era posible identificar: el ambiente de producción no es el ambiente ancestral de selección y, por lo tanto, produce un desajuste que redunda en un daño al animal. Esta (nueva) mirada desde las cuatro preguntas posibilita nuevas direcciones de investigación.

Entre esas direcciones, emerge una nueva medicina basada en la teoría de la evolución. Inicialmente en la medicina humana, ya comienzan a aparecer desarrollos que apuntan a una medicina veterinaria darwiniana (Capitelli et al., 2010).

En esta publicación hemos mostrado la aplicación de este marco conceptual a la producción porcina; resulta impensable abordar esa actualidad sin tener en cuenta el comportamiento de los animales y una de las formas de hacerlo es empleando los conceptos y métodos de la etología. Junto a una etología clínica, una etología aplicada a la producción generará el escenario no sólo para una mayor eficiencia, sino para un nuevo compromiso ético. Al (re)definir a los animales en términos de su comportamiento, se ponen en evidencia la importancia de los temas referidos al bienestar animal y sus consecuencias.

\section{Conflicto de intereses}

Todos los autores declaran que no existen conflictos de intereses, incluyendo las relaciones financieras, personales o de otro tipo con otras personas u organizaciones que pudieran influir de manera inapropiada en el trabajo.

\section{Bibliografía}

Alcock J. Proximate and ultimate causes of behavior: how and why birds sing. En: 2001. Animal behavior. An evolutionary approach. (7a ed). Massachusetts. Sinauer Associates, Inc., pp. 23-49.

Albiach-Serrano A, Bräuer J, Cacchione T, Zickert $\mathrm{N}$, Amici F. 2012. The effect of domestication and ontogeny in swine cognition (Sus scrofa scrofa and S. s. domestica). Applied Animal Behavior Science. 141:25-35.

doi: 10.1016/j.applanim.2012.07.005

Baxter MR. 1983. Ethology in environmental design for animal production. Applied Animal Ethology. 9:207-20.

doi: 10.1016/0304-3762(83)90001-9

Benson G, Rollin, B. Theoretical framework. En: 2004. The well being of farm animal, challenges and solutions. First edition. Blackwell Publishing. EE.UU, pp. 3-179. 
Broom DM. 1991. Animal welfare: Concept and measurement. Journal of Animal Science. 69:4167-75.

Capitelli GM, Ferrari HR, López H. 2010. Introducción a la medicina veterinaria Darwiniana. Aplicaciones de la teoría de la evolución en la práctica veterinaria. Vaccinología, zoonosis y bienestar animal como ejemplos de aplicación. Revista de Medicina Veterinaria (Buenos Aires). 91:(5/6)71-5.

Curio E. 1994. Causal and functional questions: how are they linked? Animal Behaviour. 47:9991021.

doi: 10.1006/anbe.1994.1139

Dewsbury DA. 1999. The proximate and the ultimate: past, present, and future. Behavioural Processes. 46:189-99.

Dostalkova W, Spinkaz M, Kindlmann P. 2002. Begging for milk: evolution of teat massaging in suckling pigs. Journal of Theoretical Biology. 215:321-32.

doi: $10.1006 /$ jtbi.2001.2511

Duncan IJ. 1995. DGM Wood-Gush Memorial Lecture: An applied ethologist looks at the question "Why?". Applied Animal Behaviour Science. 44(2-4):205-17.

doi: 10.1016/0168-1591(95)00614-X

Finlay B, Hinz F, Darlington R. 2011. Mapping behavioral evolution onto brain evolution: the strategic roles of conserved organization in individuals and species. Philosophical Transactions of the Royal Society B. 366:2111-23. doi: 10.1098/rstb.2010.0344

Fraser D, Krames DL, Pajor EA, Weary DM. 1995. Conflict and cooperation: sociobiological principles and the behaviour of pigs. Applied Animal Behavior Science. 44:139-57.

doi: 10.1016/0168-1591(95)00610-5

Fraser D, Rushen J. 1992. Calostrum intake by newborn pigs. Canadian Journal of Animal Sciences. 1-13.

doi: 10.4141/cjas92-001

Fraser D, Thompson BK. 1991. Armed sibling rivalry among suckling piglets. Behavioral Ecology and Sociobiology. 29:9-15.

Grandin T. 1980. Livestock behavior as related to handling facility design. International Journal for the Study of Animal Problems. 1:33-52.

Hartsock TG, Graves HB. 1976. Neonatal behavior and nutrition-related mortality in domestic swine. Journal of Animal Science. 42(1):235-41.
Hogan JA. 2015. A framework for the study of behavior. Behavioral Processes. 117:105-13. doi: 10.1016/j.beproc.2014.05.003

Hohenshell LM, Minick JA, Lay Jr DC, Ford SP. 1996. Maternal behavior potentially affecting offspring survivability: a comparison between Meishan and Yorkshire X Landrace (YL) sows. Swine Research Report. IOWA State University. Disponible en: http://lib.dr.iastate.edu/ swinereports [Consultado 01/01/2017].

Hoy JM, Murray PJ, Tribe A. 2010. Thirty years later: enrichment practices for captive mammals. Zoo Biology 29:303-16.

doi: 10.1002/zoo.20254

Hughes B, Duncan I. 1988. The notion of ethological 'need', models of motivation and animal welfare. Animal Behavior. 36:1696-707. doi: 10.1016/Sooo3-3472(88)80110-6

Illman G, Pokorná Z, Spinka M. 2007. Allosuckling in domestic pigs: teat acquisition strategy and consequences. Applied Animal Behavior Science. 106:26-38.

doi: 10.1016/j.applanim.2006.06.012

Jensen P. 1986. Observation of maternal behaviour of free-ranging domestic pigs. Applied Animal Behavior Science. 16:131-42.

doi: 10.1016/0168-1591(86)90105-X

Jensen P. 1993. Nest building in domestic sows: the sole of external estimuli. Animal Behavior. 45(2):351-8.

Jensen, P. Comportamiento del cerdo. 2004. Etología de los animales domésticos. Zaragoza, España. Jensen (Editor), Editorial Acribia, pp. 168-84.

Jensen P, Toates F. 1993. Who needs 'behavioural needs'? Motivational aspects of the needs of animals. Applied Animal Behavior Science. 37:161-81.

doi: 10.1016/0168-1591(93)90108-2

Lakatos I. 1983."La metodología de los programas de investigación científica". Madrid. Alianza Editorial.

Larson G. 2005. Worldwide phylogeography of wild boar reveals multiple centers of pig domestication. Science. 307:1618.

doi: 10.1126/science.1106927

Lorenz K. 1937. The companion in the bird's world. The Auk. 54:245-73.

Lorenz K. 1974. Etología comparada. El comportamiento instintivo en la filogenia. En: Biología del comportamiento. Ciudad de México; Siglo XXI Editores, pp. 7-18. 
Lorenz, Konrad. 1995. La otra cara del espejo. Plaza \& Janes.

Meunier-Salaün MC, Gort F, Prunier A, Schouten WPG. 1991. Behavioral patterns and progesterone, cortisol and prolactin levels around parturition in European (Large White) and Chinese (Meishan) sows. Applied Animal Behavior. Science. 31:43-59. doi: 10.1016/0168-1591(91)90152-N

Orihuela A, Solano JJ. 1995. Managing "teat order" in suckling pigs (Sus scrofa domestica). Applied Animal Behavior Science. 46:125-30. doi: 10.1016/0168-1591(95)00639-7

Redivatti M. 2009. Caracterización de cerdos criollos del nordeste argentino. Tesis doctoral, Universidad de Córdoba, España.

Skinner B. 1974. Sobre el conductismo. Título del original: About behaviourism. Publicado por Alfred A Knopf, New York. USA. Traducción Fernando Barrera. Ed Planeta-De Agostini Mexico DFD.

Spinka M, Algers B. 1995. Functional view on udder massage after milk let-down in pigs. Applied Animal Behavior Science. 43:197-212. doi: 10.1016/0168-1591(95)00560-F
Stamps J. 2003. Behavioral processes affecting development: Tinbergen's fourth question comes of age. Animal Behavior. 66(1):1-13.

doi: 10.1006/anbe.2003.2180

Tinbergen N. 1939. On the analysis of social organization among vertebrates, with special references to birds. The American Naturalist. 21:210-34.

Tinbergen N. 1963. "On aims and methods of ethology." Zeitschrift für Tierpsychologie. 20:41033 .

Tinbergen N. 1981. El estudio del instinto. $10^{\circ}$ edición. Ciudad de México; Siglo XXI editores.

Thorpe WH. 1965. The assessmentt of pain and distress in animals. En: Report of the Technical Committee to enquire into de welfare of animals kept under intensive livestock systems (F.W.R. Brambell). HMSO, London. UK.

Thorpe WH. 1982. Breve historia de la etología. Vol 902. Madrid, España; Alianza Editorial.

Wiepkema PR. Behavioral aspects of stress. En: 1987. Wiepkema PR; van Adrichem PWM (Editors). Biology of stress in farm animals: an integrative approach. Martinus Nijhoff, Dordrecht. 113-33. 